\title{
Multiple lung abscesses caused by Streptococcus constellatus
}

\author{
Vanina Rognoni, ${ }^{1}$ Annalisa Malara, ${ }^{2}$ Enrico Storti, ${ }^{2}$ Adriano Anesi ${ }^{1}$ \\ ${ }^{1}$ U.S.S. Microbiologia, and ${ }^{2}$ U.S.C. Terapia Intensiva e Subintensiva, ASST Lodi, Italy
}

\section{Summary}

Despite numerous descriptions of body abscesses produced by Streptococcus milleri group bacteria, lung abscesses caused by this group remain under-reported and the clinical and laboratory features have yet to be fully characterised. We present the case of a patient admitted with lung multiple abscesses produced by Streptococcus constellatus.

\section{Introduction}

The Streptococcus milleri group (Streptococcus constellatus, Streptococcus anginosus, Streptococcus intermedius) are commensal bacteria of the oropharyngeal, gastrointestinal and genital flora, which have been identified over the last few decades as a cause of purulent infections in various parts of the body (6). Cases of lung abscesses are rarely reported in the literature but known predisposing factors include inhalation, chronic or malig-

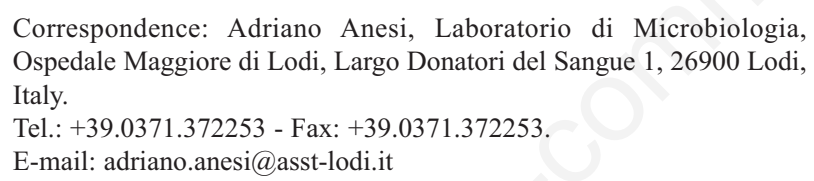

Key words: Streptococcus constellatus; Streptococcus milleri group, lung abscess; pneumonia.

Contributions: VR and AA: design of the work, acquisition and interpretation of data for the work, drafting the work; AM and ES: acquisition and interpretation of data for the work, revising the work critically for important intellectual content.

Conflict of interest: the authors declare no potential conflict of interest.

Received for publication: 20 September 2017.

Revision received: 5 January 2018.

Accepted for publication: 5 January 2018

(C) Copyright V. Rognoni et al., 2017

Licensee PAGEPress, Italy

Microbiologia Medica 2017; 32:7082

doi:10.4081/mm.2017.7082

This article is distributed under the terms of the Creative Commons Attribution Noncommercial License (by-nc 4.0) which permits any noncommercial use, distribution, and reproduction in any medium, provided the original author(s) and source are credited. nant lung diseases, diabetes mellitus, alcohol abuse, uncontrolled epilepsy, and periodontal diseases (3). Here we present the case of a young man with multiple lung abscesses caused by Streptococcus constellatus.

\section{Case Report}

A 49-year-old man was taken to the A\&E department of the Maggiore Hospital in Lodi, suffering from dyspnoea, fever and lethargy. His anamnesis revealed moderate mental retardation with behavioural abnormalities, coupled with long-term consumption of barbiturates, antipsychotics and anticonvulsants. The day before admission he took a single dose of levofloxacin of $500 \mathrm{mg}$ per os on advice from his physician.

Laboratory testing revealed an initial white blood cell count (WBC) of $21.06 \times 10^{3} / \mathrm{uL}$ (normal range, $4.0-10.0 \times 10^{3} / \mathrm{uL}$ ) with a differential of $89.3 .6 \%$ neutrophils $(40-74 \%)$, haemoglobin of $11.4 \mathrm{~g} / \mathrm{dL}(14-18 \mathrm{~g} / \mathrm{dL})$ and a platelet count of $457 \times 10^{3} / \mathrm{uL}(130-$ $\left.400 \times 10^{3} / \mathrm{uL}\right)$. Measurement of inflammatory markers showed $\mathrm{C}$ reactive protein levels of $400 \mathrm{mg} / \mathrm{L}(0.0-7.5 \mathrm{mg} / \mathrm{L})$ and procalcitonin levels of $10.35 \mathrm{ng} / \mathrm{mL}(0.0-0.5 \mathrm{ng} / \mathrm{mL})$.

A room air $\mathrm{SpO}_{2}$ value of $86 \%$ was recorded; this value increased to $91 \%$ when oxygen was administered at a flow rate of 7 litres per minute.

His chest X-ray showed parenchymal thickening on the left lobe. Hence, the patient was admitted to the general ward for pneumonia and was treated with levofloxacin $(500 \mathrm{mg}$ intravenously every 24 hours) and ceftriaxone ( $2 \mathrm{~g}$ intravenously every 12 hours).

Due to a deterioration of his clinical condition, the patient was transferred to the Intensive Care Unit on the second day, where he was intubated and mechanically ventilated for the following five days. The antibiotic therapy was escalated by adding a continuous infusion of linezolid $1200 \mathrm{mg} /$ day. His chest CT scan showed two large abscesses in the left lung and a third one in the right lung (Figure 1). Bronchoalveolar lavage (BAL) fluid samples were collected for microbiological investigations. Direct microscopic examination after Gram staining of the material showed the presence of a layer of polymorphonuclear leukocytes and numerous Gram-positive cocci in chains, most of which were intracellular (Figure 2). Filamentous, branching Gram-positive structures suspected to be Actimomyces or Nocardia species were not observed and consequently no specific cultures were performed. Acid-fast staining, nested-PCR (Xpert MTB/RIF Assay, Cepheid. Sunnyvale, CA, USA) and cultures in liquid (BD Bactec MGIT, Becton Dickinson, Sparks, MD, USA) and solid media were negative for Mycobacteria. Quantitative cultures were performed on BAL fluid samples, and organisms were identified when present in significant amounts $\left(\geq 10^{4} \mathrm{CFU} / \mathrm{mL}\right)$ (2). BAL fluid cultures were incubated at $35^{\circ} \mathrm{C}$ to $37^{\circ} \mathrm{C}$ both in $5 \% \mathrm{CO}_{2}$ and in ambient air for 48 hours. For the cultivation of anaerobic bacteria BAL fluid cul- 
tures were incubated at $35^{\circ} \mathrm{C}$ to $37^{\circ} \mathrm{C}$ using the GasPaK EZ Gas Generating Pouch System (Becton Dickinson, Sparks, MD, USA). The primary culture from BAL fluid contained just one species of microorganism, identified as Streptococcus constellatus by Vitek MS MALDI-TOF mass spectrometry instrument (bioMerieux, Marcy-l'Etoile, France).

Antibiotic susceptibility tests, performed by E-test (bioMérieux) according to EUCAST, indicated susceptibility to: penicillin (MIC 0.125), amoxicillin/clavulanic acid (MIC 0.25), piperacillin/tazobactam (MIC 0.25), imipenem (MIC 0.125), meropenem (MIC 0.64), levofloxacin (MIC 0.25), vancomycin (MIC 1), linezolid (MIC 0.5), clindamycin (MIC 0.125) and resistance to metronidazole ( $\mathrm{MIC} \geq 256$ ). On the fourth day, following a steady improvement in the patient's condition and in the light of the antibiogram results, antibiotic treatment was de-escalated switching to amoxicillin/clavulanate ( $2.2 \mathrm{~g}$ intravenously every 8 hours for 10 days). A chest CT scan carried out on the tenth day showed a reduction of the parenchymal thickening, albeit without a complete restitutio ad integrum of the parenchyma involved.
Bronchoalveolar lavage cultures performed on the eleventh day were negative. The patient was therefore transferred to the Respiratory Medicine ward for ongoing treatment and later discharged after a two-week hospital stay.

\section{Conclusions}

Lung abscesses present as circumscribed accumulations of pus in the lungs and are usually a complication of aspiration pneumonia. More often than not, the aetiology is due to anaerobic bacteria found in the oral cavity microflora, such as the Peptostreptococcus, Bacteroides, and Fusobacterium species. Less frequently, it is possible to encounter aerobic bacteria such as $S$. aureus, S. pyogenes, S. pneumoniae, $H$. influenzae, K. pneumoniae, Nocardia species and Actinomyces species (5).

Streptococcus constellatus is increasingly being recognised as an important pulmonary pathogen which may lead to the develop-
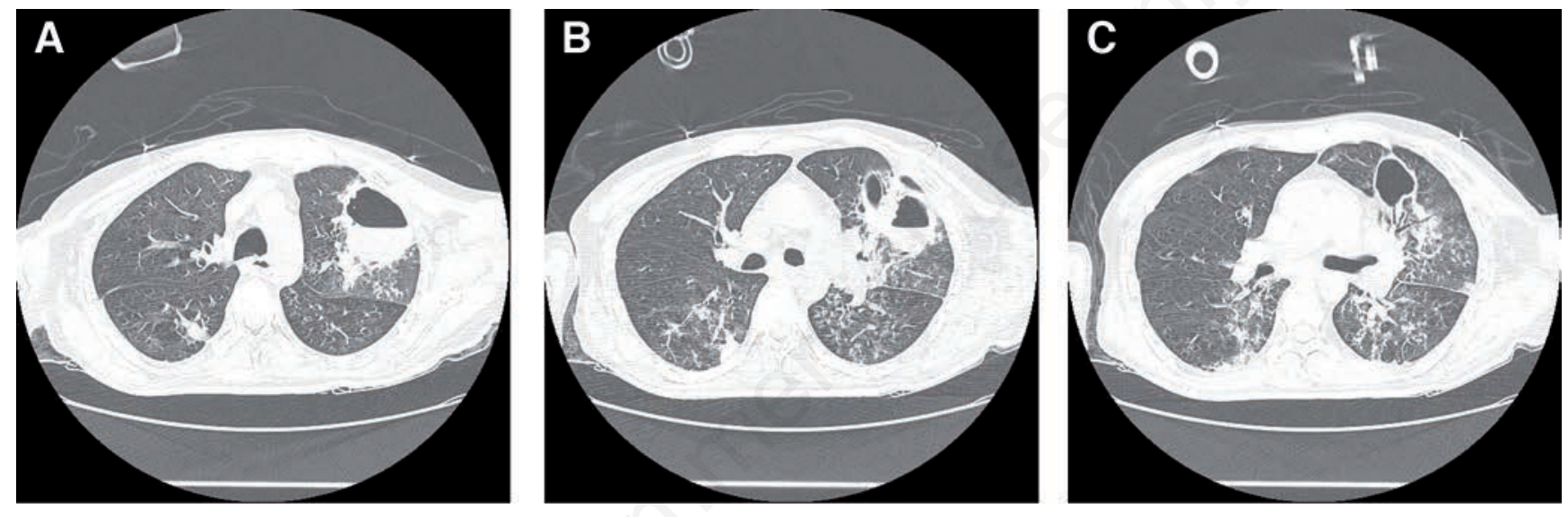

Figure 1. A) Chest computed tomogram carried out on the second day. In the left upper pulmonary lobe two large excavated lesions are found side-by-side, each with a bronchial structure, one of which is clear, the other distinctly showing an air-fluid level, characterized by rather thin walls. B) In the posterior basal segment of the ipsilateral lower lobe, an extended area of parenchymal consolidation can be observed, with evidence of another smaller excavated lesion with an air-fluid level. C) In the right upper lobe posterior segment and left upper lobe apicoposterior segment, as well as in the apical and posterior basal segments of the lobes on both sides, computed tomogram shows multiple areas of parenchymal consolidation, with more extensive areas on the left.
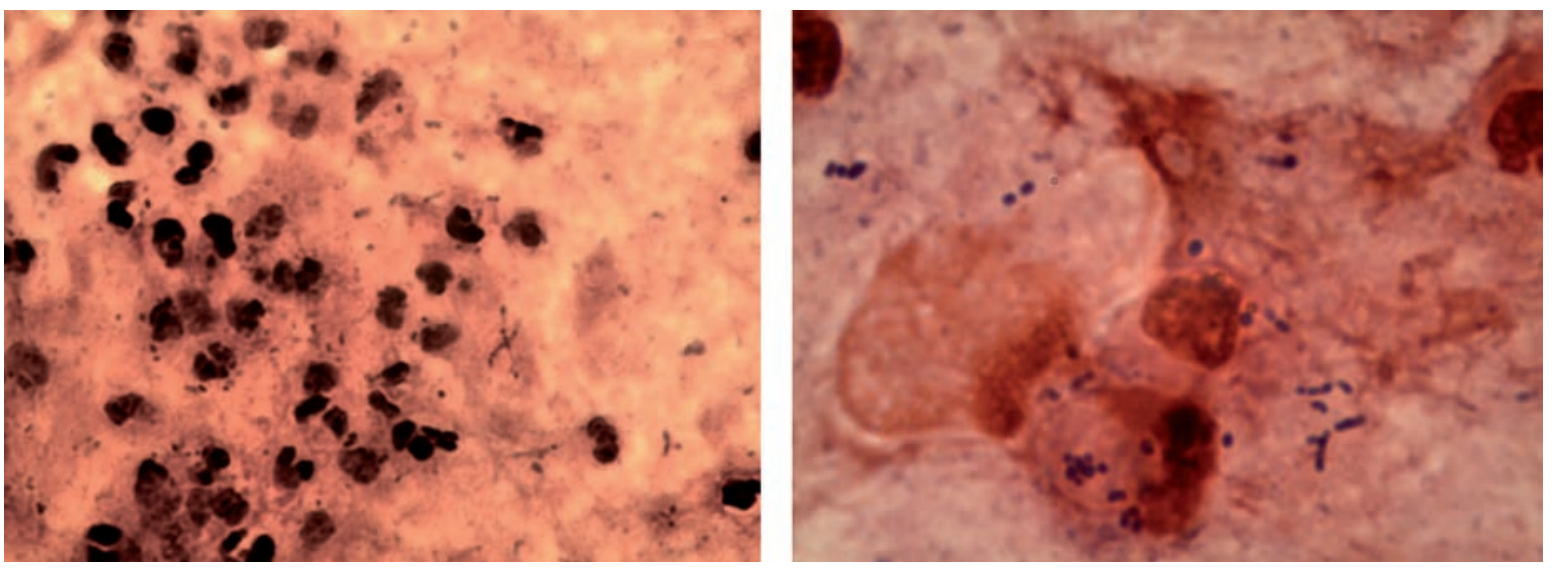

Figure 2. Direct microscopic examination of bronchoalveolar lavage cells after Gram staining (400× and 1000× magnification). 
ment of empyema or lung abscess, although just a few small series have been reported in the literature $(1,4)$. A retrospective review of 25 cases of empyema and/or lung abscesses caused by the Streptococci milleri group shows significant morbidity and mortality, with a higher incidence in males (7). These microorganisms are susceptible to all $\beta$-lactams, while they present varying levels of susceptibility to tetracycline, clindamycin and erythromycin. Conservative therapy with administration of antibiotics, such as in the clinical case presented here, is usually curative. For more severe cases, surgical drainage is required.

\section{References}

1. Gogineni VK, Modrykamien A. Lung abscesses in 2 patients with Lancefield group F streptococci (Streptococcus milleri group). Respir Care 2011;56:1966-9.
2. Leber AL, ed. Lower respiratory tract cultures. In: Clinical Microbiology Procedures Handbook. 4th ed. Washington DC: American Society for Microbiology Press; 2016.

3. Noguchi S, Yatera K, Kawanami T, et al. The clinical features of respiratory infections caused by the Streptococcus anginosus group. BMC Pulmon Med 2015;15:133.

4. Shinzato T, Saito A. The Streptococcus milleri group as a cause of pulmonary infection. Clin Infect Dis 1995;21:S238-43.

5. Takayanagi N, Kagiyama N, Ishiguro T, et al. Etiology and outcome of community-acquired lung abscess. Respiration 2010;80:98-105.

6. Whiley RA, Beighton D, Winstanley TG, et al. Streptococcus intermedius, Streptococcus constellatus, and Streptococcus anginosus (the Streptococcus milleri group): association with different body sites and clinical infections. J Clin Microbiol 1992;30:243-4.

7. Wong CA, Donald F, Macfarlane JT. Streptococcus milleri pulmonary disease: a review and clinical description of 25 patients. Thorax 1995;50:1093-96. 